\title{
Medium-heavy nuclei from nucleon-nucleon interactions in lattice QCD
}

\section{AUTHOR(S):}

Inoue, Takashi; Aoki, Sinya; Charron, Bruno; Doi, Takumi; Hatsuda, Tetsuo; Ikeda, Yoichi; Ishii, Noriyoshi; Murano, Keiko; Nemura, Hidekatsu; Sasaki, Kenji

\section{CITATION:}

Inoue, Takashi ... [et al]. Medium-heavy nuclei from nucleon-nucleon interactions in lattice QCD. Physical Review C - Nuclear Physics 2015, 91(1): 011001(R).

\section{ISSUE DATE:}

2015-01-16

URL:

http://hdl.handle.net/2433/250358

\section{RIGHT:}

(c) 2015 American Physical Society. 


\title{
Medium-heavy nuclei from nucleon-nucleon interactions in lattice QCD
}

\author{
Takashi Inoue, ${ }^{1}$ Sinya Aoki, ${ }^{2,3}$ Bruno Charron, ${ }^{4,5}$ Takumi Doi, ${ }^{4}$ Tetsuo Hatsuda,,${ }^{4,6}$ Yoichi Ikeda, ${ }^{4}$ Noriyoshi Ishii, \\ Keiko Murano, ${ }^{7}$ Hidekatsu Nemura ${ }^{3}$ and Kenji Sasaki ${ }^{3}$ \\ (HAL QCD Collaboration) \\ ${ }^{1}$ Nihon University, College of Bioresource Sciences, Kanagawa 252-0880, Japan \\ ${ }^{2}$ Yukawa Institute for Theoretical Physics, Kyoto University, Kyoto 606-8502, Japan \\ ${ }^{3}$ Center for Computational Sciences, University of Tsukuba, Ibaraki 305-8571, Japan \\ ${ }^{4}$ Theoretical Research Division, Nishina Center, RIKEN, Saitama 351-0198, Japan \\ ${ }^{5}$ Department of Physics, University of Tokyo, Tokyo 113-0033, Japan \\ ${ }^{6}$ Kavli IPMU (WPI), University of Tokyo, Chiba 277-8583, Japan \\ ${ }^{7}$ Research Center for Nuclear Physics (RCNP), Osaka University, Osaka 567-0047, Japan
}

(Received 1 September 2014; published 16 January 2015)

\begin{abstract}
On the basis of the Brueckner-Hartree-Fock method with the nucleon-nucleon forces obtained from lattice QCD simulations, the properties of the medium-heavy doubly magic nuclei such as ${ }^{16} \mathrm{O}$ and ${ }^{40} \mathrm{Ca}$ are investigated. We found that those nuclei are bound for the pseudoscalar meson mass $M_{\mathrm{PS}} \simeq 470 \mathrm{MeV}$. The mass number dependence of the binding energies, single-particle spectra, and density distributions are qualitatively consistent with those expected from empirical data at the physical point, although these hypothetical nuclei at heavy quark mass have smaller binding energies than the real nuclei.
\end{abstract}

DOI: 10.1103/PhysRevC.91.011001

PACS number(s): 12.38.Gc, 13.75.Cs, 21.10.-k

Studying the ground and excited states of finite nuclei and nuclear matter on the basis of the quantum chromodynamics (QCD) has been one of the greatest challenges in modern nuclear physics. Thanks to the recent advances in lattice QCD, we now have two major approaches to attacking this long-standing problem: The first approach is to simulate finite nuclei (systems with total baryon number $A$ ) directly on the lattice $[1,2]$. The second approach is to calculate the properties of finite nuclei and nuclear matter by using nuclear many-body techniques combined with the nuclear forces obtained from lattice QCD [3]. There is also a third approach where nuclear many-body techniques are combined with the nuclear forces from chiral perturbation theory (see, e.g., [4] and references therein); it has a close connection with the second approach through the short distance part of the nuclear forces.

In this article, we will report a first exploratory attempt to study the structure of medium-heavy nuclei $\left({ }^{16} \mathrm{O}\right.$ and $\left.{ }^{40} \mathrm{Ca}\right)$ on the basis of the second approach by HAL QCD Collaboration [3]. Before going into the detail, let us first summarize several limitations of the first approach (direct QCD simulations of finite nuclei): (i) The number of quark contractions sharply increases for larger $A$, which makes the calculation prohibitively expensive. Even with the help of newly discovered contraction algorithms [5], it is still unrealistic to make simulations for medium-heavy nuclei with controlled $S / N$ on lattice. (ii) The energy difference between the ground state and excited states, $\triangle E$, is about the QCD scale $(\sim 200 \mathrm{MeV})$ for single hadrons, while it becomes $O(10)-O(100)$ times smaller for finite nuclei, which implies that extremely large Euclidean time $t \simeq 1 / \Delta E \sim 100 \mathrm{fm}$ or more is necessary to obtain sensible nuclear spectra. (iii) The larger spatial lattice volume $V$ becomes necessary for larger nuclei. This poses a challenge particularly for heavy nuclei and/or neutron-rich nuclei. (iv) Analyzing the detailed spatial structure of nuclei (e.g., the $3 \alpha$ configuration of the Hoyle state of ${ }^{12} \mathrm{C}$ known to be crucial for the stellar nucleosynthesis) requires much more efforts beyond the calculation of binding energies.

The basic strategy of the second approach is to start with the lattice QCD simulations of nuclear forces in the form of the $A$-body potentials $(A=2,3, \ldots)$. The nuclear structures can then be calculated by the nuclear many-body techniques with the simulated potentials as inputs. This two-step approach with the "potential" (the interaction kernel) as an intermediate tool provides not only a close link to the traditional nuclear physics but also a clue to overcoming the limitations (i)-(iv) mentioned above: (i) The effect of the $A$-body potentials would decrease as $A$ increases for finite nuclei, since the empirical saturation density $\rho_{0}=0.16 / \mathrm{fm}^{3}$ is rather low. Then, we can focus mainly on the 2-body, 3-body, and possibly 4-body potentials, exploiting the modern contraction algorithm [5]. (ii) Separation of the ground state and the excited states is not necessarily to obtain the potentials as long as the system is below the pion production threshold [3]. In other words, all of the information for $t>1 \mathrm{fm}$ outside the range of inelastic region can be used to extract the potentials. (iii) The potentials among nucleons are always short ranged independent of $A$, so that they are insensitive to the lattice volume [6]. (iv) Once the potentials in the continuum and infinite volume limit are obtained, various observables can be obtained, e.g., the scattering phase shifts, the nuclear binding energies, level structures, density distributions, etc.

As a first exploratory attempt, we limit ourselves to the two-body potentials in the $S$ and $D$ waves in this article to study the structure of ${ }^{16} \mathrm{O}$ and ${ }^{40} \mathrm{Ca}$. These potentials were previously obtained in Ref. [7] where the Nambu-Bethe-Salpeter (NBS) wave functions between two baryons simulated on the lattice are translated into the two-body potentials on the basis of the HAL QCD method (reviewed in the last reference of [3]). The resultant potentials in the nucleon-nucleon channel were 
TABLE I. Masses of pseudoscalar meson $M_{\mathrm{PS}}$, vector meson $M_{\mathrm{V}}$, and octet baryon $M_{\mathrm{B}}$ in our calculation taken from [7]. Statistical error is given in parentheses.

\begin{tabular}{lrc}
\hline \hline$M_{\mathrm{PS}}(\mathrm{MeV})$ & $M_{\mathrm{V}}(\mathrm{MeV})$ & $M_{\mathrm{B}}(\mathrm{MeV})$ \\
\hline $1170.9(7)$ & $1510.4(0.9)$ & $2274(2)$ \\
$1015.2(6)$ & $1360.6(1.1)$ & $2031(2)$ \\
$836.5(5)$ & $1188.9(0.9)$ & $1749(1)$ \\
$672.3(6)$ & $1027.6(1.0)$ & $1484(2)$ \\
$468.6(7)$ & $829.2(1.5)$ & $1161(2)$ \\
\hline \hline
\end{tabular}

applied to ${ }^{4} \mathrm{He}$ with stochastic variational method in Ref. [7] and to nuclear matter with Brueckner-Hartree-Fock (BHF) method in Ref. [8].

We employ the standard BHF theory to calculate finite nuclei [9]: The main reason is that the BHF theory is simple but quantitative enough to grasp the essential part of physics, so that it is a good starting point before making precise calculations using sophisticated $a b$ initio methods such as the Green's function Monte Carlo method [10], no-core shell model [11,12], coupled-cluster theory [13], unitary-model-operator approach [14], self-consistent Green's function method [15], and in-medium similarity renormalization group approach [16].

Let us briefly recapitulate the basic equations in the BHF theory for finite nuclei to set our notation. The effective nucleon-nucleon interaction is dictated by the $G$ matrix satisfying the Bethe-Goldstone equation

$$
G(\omega)_{i j, k l}=V_{i j, k l}+\frac{1}{2} \sum_{m, n}^{\text {un-occ }} \frac{V_{i j, m n} G(\omega)_{m n, k l}}{\omega-e_{m}-e_{n}+i \epsilon},
$$

where indices $i$ to $n$ stand for single-particle eigenstates, $V$ is the bare $N N$ potential, and the sum is taken for un-occupied states. Given $G$, the single-particle potential $U$ is written as $U_{a b}=\sum_{c, d} G(\tilde{\omega})_{a c, b d} \rho_{d c}$, where the indices $a, b, c, d$ are the labels for the harmonic-oscillator (HO) basis. The density matrix $\rho$ in this basis is given by $\rho_{a b}=\sum_{i}^{\mathrm{occ}} \Psi_{a}^{i} \Psi_{b}^{i *}$, where $\Psi^{i}$ is a solution of the Hartree-Fock equation,

$$
[K+U] \Psi^{i}=e_{i} \Psi^{i},
$$

with $K$ being the kinetic energy operator. After determining $G, U, \rho, \Psi^{i}$, and $e_{i}$ self-consistently, the ground state energy of a nucleus is obtained as

$$
E_{0}=\sum_{a, b}\left[K_{a b}+\frac{1}{2} U_{a b}\right] \rho_{b a}-K_{\text {c.m. }} .
$$

Here $K_{\mathrm{c} . \mathrm{m}}$. corresponds to the subtraction of the spurious center-of-mass motion.

For the bare $N N$ potentials to be used in Eq. (1), we adopt those obtained on a $(4 \mathrm{fm})^{3}$ lattice with five different quark masses in the flavor-SU(3) limit [7] as summarized in Table I. As shown in Fig. 1, the lattice $N N$ potentials in $S$ and $D$ waves at the pseudoscalar meson mass $M_{\mathrm{PS}} \simeq 470 \mathrm{MeV}$ share common features with phenomenological potentials, i.e., a strong repulsive core at short distance, an attractive pocket at intermediate distance, and a strong ${ }^{3} S_{1}-{ }^{3} D_{1}$ coupling. Although

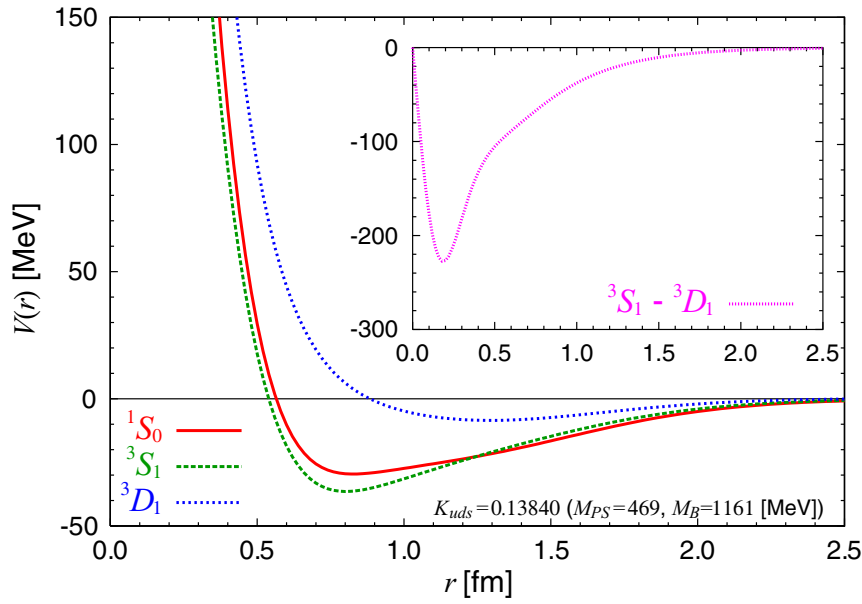

FIG. 1. (Color online) Nucleon-nucleon potentials for $S$ and $D$ waves in lattice QCD at $M_{\mathrm{PS}} \simeq 470 \mathrm{MeV}$. The lines are obtained by the least-chi-square fit to the lattice data.

the potentials reproduce qualitative features of experimental phase shifts, the net attraction is still too weak to form a deuteron bound state [7], while it is strong enough to have saturation of symmetry nuclear matter (SNM) [8].

Using these lattice $N N$ potentials, together with the nucleon mass, as inputs, we carry out the BHF calculation for the ground states of ${ }^{16} \mathrm{O}$ and ${ }^{40} \mathrm{Ca}$ nuclei. We choose these nuclei since they are isosymmetric, doubly magic, and spin saturated, and hence we can assume spherically symmetric nucleon distribution. Due to the limitation of available lattice $N N$ potentials at present, we include 2-body $N N$ potentials only in ${ }^{1} S_{0},{ }^{3} S_{1}$, and ${ }^{3} D_{1}$ channels. The Coulomb force between protons is not taken into account for simplicity. We follow Refs. [17,18] about the numerical procedure of BHF calculation, i.e., we solve Eq. (1) by separating the relative and center-of-mass coordinates using the Talmi-Moshinsky coefficient, and adopt the so-called $Q /(\omega-Q K Q) Q$ choice, where $Q$ is the Pauli exclusion operator for which we use a harmonic-oscillator one at first then use a self-consistent one for the last few iterations. In Eq. (3), the center-of-mass correction is estimated as $K_{\text {c.m. }} \simeq \frac{3}{4} \hbar \omega$ with $\omega$ being the a HO frequency which reproduces the rms radius of the matter distribution obtained by the BHF calculation.

Figure 2 shows the ground state energy of ${ }^{16} \mathrm{O}$ at $M_{\mathrm{PS}} \simeq$ $470 \mathrm{MeV}$, as a function of the width parameter $b$ of the $\mathrm{HO}$ wave function with increasing number of $\mathrm{HO}$ basis $n_{\mathrm{dim}}$. The solid vertical bar at the rightmost point represents the error for $E_{0}$ of about $\pm 10 \%$ at $b=3 \mathrm{fm}$ and $n_{\mathrm{dim}}=9$. It originates from the statistical error of our lattice QCD simulations estimated by the jackknife analysis with the bin size of 360 for 720 measurements as was done in Ref. [8]. Almost the same errors apply to other $E_{0}$ in the figure. A similar figure for ${ }^{40} \mathrm{Ca}$ is obtained for the same quark mass. As $n_{\text {dim }}$ increases, the binding energy $\left|E_{0}\right|$ increases with the optimal $b$ shifting to larger values. From these results, we can definitely say that self-bound systems are formed in both nuclei at this lightest quark mass, corresponding to $M_{\mathrm{PS}} \simeq 470 \mathrm{MeV}$ and $M_{\mathrm{B}} \simeq 1160 \mathrm{MeV}$. On the other hand, the existence of deeply 


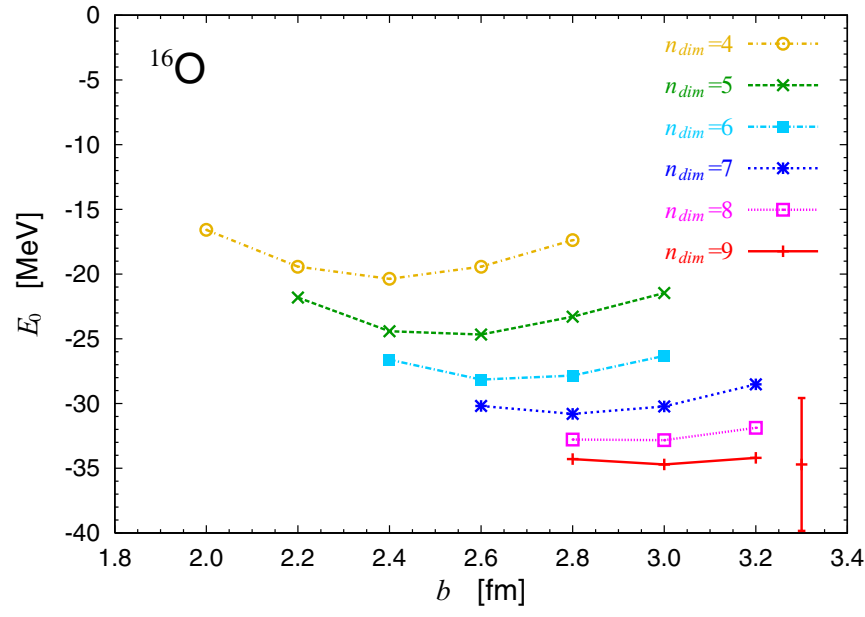

FIG. 2. (Color online) Ground state energy of ${ }^{16} \mathrm{O}$ at $M_{\mathrm{PS}} \simeq$ $470 \mathrm{MeV}$ as a function of $b$ at several $n_{\mathrm{dim}}$.

bound nuclei is excluded for the other four heavier quark masses, since we do not find $E_{0}<0$.

In Figure 3, single-particle levels of ${ }^{16} \mathrm{O}$ and ${ }^{40} \mathrm{Ca}$ at $M_{\mathrm{PS}} \simeq$ $470 \mathrm{MeV}$ are shown for the optimal width parameter with the largest $\mathrm{HO}$ basis; $b=3.0 \mathrm{fm}$ and $n_{\mathrm{dim}}=9$. In spite of the unphysical quark mass in our lattice QCD simulations, the obtained single-particle levels have the similar magnitude expected for those nuclei in the real world. Also, in the bound region, the level structure follows almost exactly the harmonic oscillator spectra with $\hbar \omega \simeq 22-23 \mathrm{MeV}$. Since the spin-orbit force is not included in our lattice nuclear force, the spin-orbit splittings in the $P$ and $D$ states are not seen in the figure.

Table II shows the single-particle energies, total binding energies, and rms radii of the matter distributions of ${ }^{16} \mathrm{O}$ and ${ }^{40} \mathrm{Ca}$ at $M_{\mathrm{PS}} \simeq 470 \mathrm{MeV}$ for $b=3.0 \mathrm{fm}$ and $n_{\mathrm{dim}}=9$. Breakdowns of the total binding energies are

$$
\begin{aligned}
{ }^{16} \mathrm{O}: E_{0} & =(259.6-10.3)-284.0=-34.7 \mathrm{MeV}, \\
{ }^{40} \mathrm{Ca}: E_{0} & =(813.4-9.8)-916.3=-112.7 \mathrm{MeV},
\end{aligned}
$$

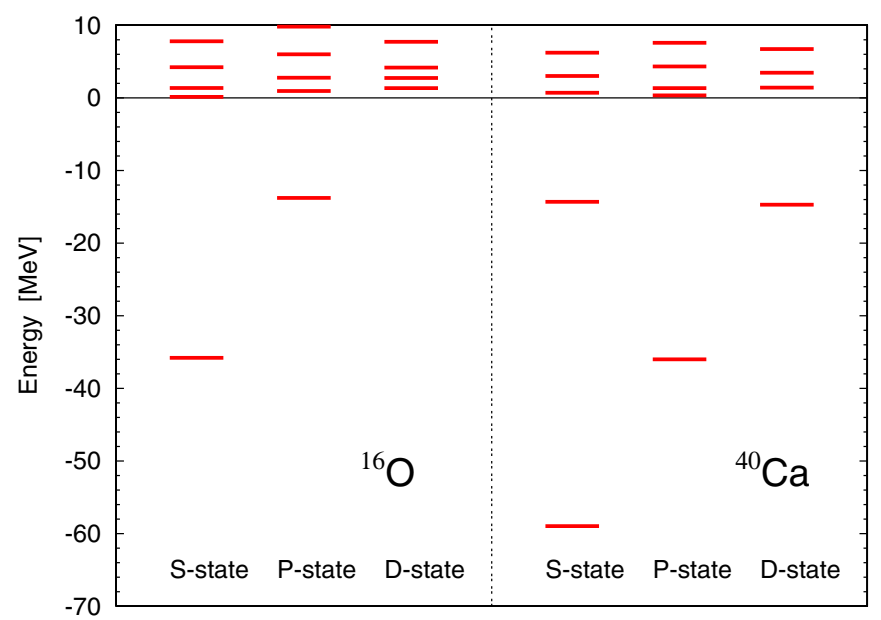

FIG. 3. (Color online) Single-particle levels of ${ }^{16} \mathrm{O}$ and ${ }^{40} \mathrm{Ca}$ nuclei at $M_{\mathrm{PS}} \simeq 470 \mathrm{MeV}$. Positive energy continuum states appear as discrete levels due to the finite number of bases.
TABLE II. Single-particle levels, total energy, and rms radius of ${ }^{16} \mathrm{O}$ and ${ }^{40} \mathrm{Ca}$ at $M_{\mathrm{PS}} \simeq 470 \mathrm{MeV}$. Energies (radii) are in unit of $\mathrm{MeV}$ (fm).

\begin{tabular}{lccccccccc}
\hline \hline & \multicolumn{3}{c}{ Single-particle level } & & \multicolumn{2}{c}{ Total energy } & Radius \\
\cline { 2 - 3 } & $1 S$ & $1 P$ & $2 S$ & $1 D$ & & $E_{0}$ & $E_{0} / A$ & $\sqrt{\left\langle r^{2}\right\rangle}$ \\
\hline${ }^{16} \mathrm{O}$ & -35.8 & -13.8 & & & & -34.7 & -2.17 & 2.35 \\
${ }^{40} \mathrm{Ca}$ & -59.0 & -36.0 & -14.7 & -14.3 & -112.7 & -2.82 & 2.78 \\
\hline \hline
\end{tabular}

where the first, second, and third numbers are the kinetic energy, the center-of-mass correction, and the potential energy, respectively. The total binding energy is obtained as a result of a large cancellation between kinetic energy and potential energy. Principally due to the heavier quark mass in our calculation, the obtained binding energies $\left|E_{0}\right|$ are smaller than the experimental data, $127.6 \mathrm{MeV}$ for ${ }^{16} \mathrm{O}$ and $342.0 \mathrm{MeV}$ for ${ }^{40} \mathrm{Ca}[19]$.

The rms radii of the matter distribution given in Table II are calculated without the nucleon form factor and the centerof-mass correction. We found that these radii are more or less similar to experimental charge radii $\left(2.73 \mathrm{fm}\right.$ for ${ }^{16} \mathrm{O}$ and $3.48 \mathrm{fm}$ for ${ }^{40} \mathrm{Ca}$ ), although our quark mass is heavier. This is presumably due to a cancellation between heavier nucleons and weaker nuclear forces than in the real world. Shown in Fig. 4 is the spatial distribution of baryon number density $\rho(r)$ for ${ }^{16} \mathrm{O}$ and ${ }^{40} \mathrm{Ca}$ as a function of the distance from the center of the nucleus. The bump and dent at small distance originate from the shell structures which are known to exist in the nuclear charge distribution extracted from the electronnucleus scattering experiments. We also find that the central baryon density is as high as $2 \rho_{0}$ for ${ }^{40} \mathrm{Ca}$. This is consistent with the fact that the saturation density of SNM for the present quark mass with 2-body $N N$ forces is about $2.5 \rho_{0}$ [8].

Finally, in Fig. 5, the binding energies per particle $E_{0} / A$ for $A=4,16,40$, and $\infty$ obtained by using the same lattice potential at $M_{\mathrm{PS}} \simeq 470 \mathrm{MeV}$ are plotted as a function of

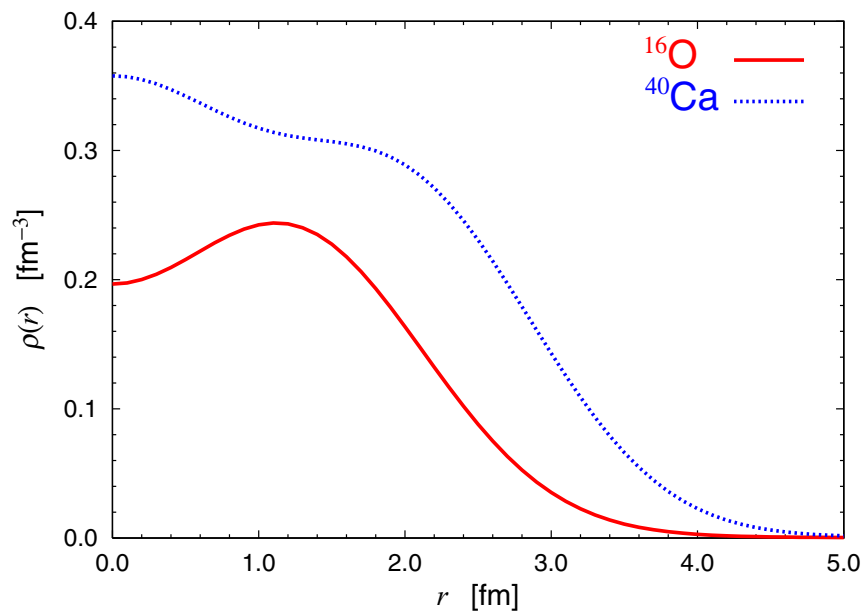

FIG. 4. (Color online) Nucleon number density inside ${ }^{16} \mathrm{O}$ and ${ }^{40} \mathrm{Ca}$ at $M_{\mathrm{PS}} \simeq 470 \mathrm{MeV}$ as a function of distance from the center of the nucleus. 


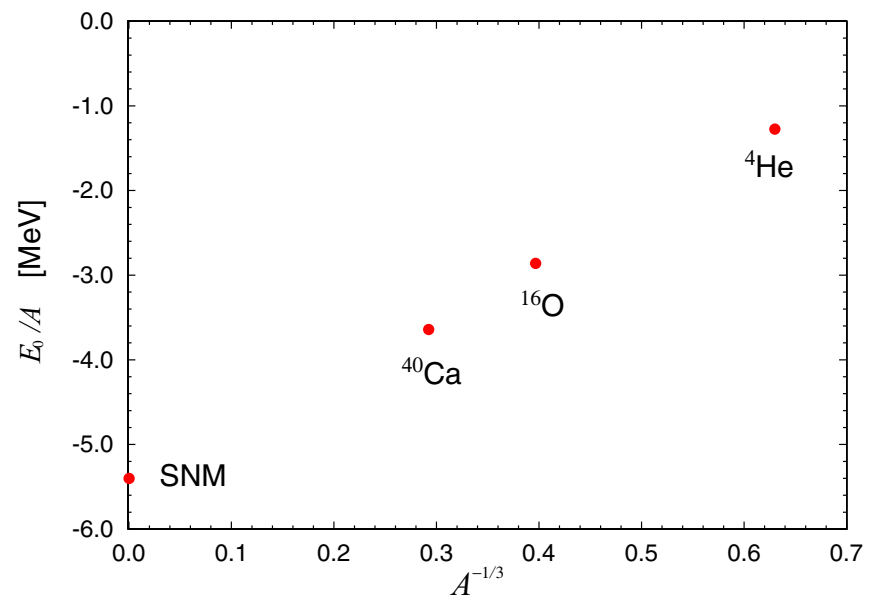

FIG. 5. (Color online) Mass number $A$ dependence of nuclear energy per nucleon $E_{0} / A$ for $M_{\mathrm{PS}} \simeq 470 \mathrm{MeV}$. The Bethe-Weizsäcker mass formula up to the second term, $E_{0} / A=-a_{\mathrm{V}}-a_{\mathrm{S}} A^{-1 / 3}$, corresponds to a straight line in this figure.

$A^{-1 / 3}$. The stochastic variational method is used for ${ }^{4} \mathrm{He}$ [7], while the BHF method is used for SNM [8]. To make a fair comparison to these cases, we carry out a linear extrapolation of the binding energies of ${ }^{16} \mathrm{O}$ and ${ }^{40} \mathrm{Ca}$ to $n_{\mathrm{dim}}=\infty$ through the formula $E_{0}\left(A ; n_{\text {dim }}\right)=E_{0}(A ; \infty)+c(A) / n_{\text {dim }}$. The linear formula fits our results well, although the convergence to $n_{\mathrm{dim}}=\infty$ is relatively slow. (The faster convergence may be achieved by employing the approaches such as $V_{\text {low } k}$ and the similarity renormalization group [13]). Our procedure leads to $E_{0}(16 ; \infty) / 16=-2.86 \mathrm{MeV}$ and $E_{0}(40 ; \infty) / 40=$ $-3.64 \mathrm{MeV}$. Note that these numbers are subject to the $\pm 10 \%$ uncertainty due to the statistical error in the $N N$ interactions from lattice QCD as mentioned already. Although the magnitude of $\left|E_{0} / A\right|$ for ${ }^{16} \mathrm{O},{ }^{40} \mathrm{Ca}$, and SNM are a factor of $3-4$ smaller than the empirical values, its $A$ dependence is uniform and can be approximated by the Bethe-Weizsäcker type mass formula, $E_{0}(A)=-a_{\mathrm{V}} A-a_{\mathrm{S}} A^{2 / 3}$, with $a_{\mathrm{V}}=5.46 \mathrm{MeV}$ and
$a_{\mathrm{S}}=-6.56 \mathrm{MeV}$. It would be interesting in the future to study the quark mass dependences of $a_{\mathrm{V}, \mathrm{S}}$ in the lighter quark mass region and investigate how these coefficients approach the empirical values, $a_{\mathrm{V}}^{\text {phys }}=15.7 \mathrm{MeV}$ and $a_{\mathrm{S}}^{\text {phys }}=-18.6 \mathrm{MeV}$.

In this Rapid Communication, we have shown that properties of medium-heavy nuclei can be deduced by combining the nuclear many-body method with the nuclear force obtained from lattice QCD simulations. Using the BHF theory with 2-body $N N$ potentials at $M_{\mathrm{PS}} \simeq 470 \mathrm{MeV}$, we found bound nuclei for ${ }^{16} \mathrm{O}$ and ${ }^{40} \mathrm{Ca}$, and we could extract their binding energies, single-particle spectra, and density distributions. Even though our setup is still primitive in various places, our results demonstrate that the HAL QCD approach to nuclear physics is quite promising for unraveling the structure of finite nuclei and infinite nuclear matter in a unified manner from QCD.

In the present study, we have neglected the nuclear forces in $P, F$ and higher partial waves, in particular the effect of the spin-orbit $(L S)$ force: For nuclei with $A>40$, the $L S$ force plays a crucial role in developing the magic numbers. Therefore it will be an important next step to include the $L S$ force recently extracted from lattice QCD simulations [20]. The 3-body force may also play an essential role for accurate determinations of the binding energy and the structure of finite nuclei as well as nuclear matter. Study of the three-nucleon force in QCD is also in progress [21]. Finally, the masses of up and down quarks in this study are much heavier than the physical values. We are currently working on the almost physical point lattice QCD simulations with the lattice volume $(8 \mathrm{fm})^{3}$ on the K-computer at RIKEN AICS. Lattice QCD potentials obtained in such simulations together with advanced nuclear many-body methods will open a new connection between QCD and nuclear physics.

This research is supported in part by Grant-in-Aid of MEXT-Japan for Scientific Research (B) 25287046, 24740146, (C) 26400281, 23540321, and SPIRE (Strategic Program for Innovative REsearch). T.H. was partially supported by RIKEN iTHES Project.
[1] T. Yamazaki, Y. Kuramashi, and A. Ukawa (PACS-CS Collaboration), Phys. Rev. D 81, 111504 (2010); T. Yamazaki, K. I. Ishikawa, Y. Kuramashi, and A. Ukawa, ibid. 86, 074514 (2012).

[2] S. R. Beane et al. (NPLQCD Collaboration), Phys. Rev. D 85, 054511 (2012); 87, 034506 (2013).

[3] N. Ishii, S. Aoki, and T. Hatsuda, Phys. Rev. Lett. 99, 022001 (2007); S. Aoki, T. Hatsuda, and N. Ishii, Prog. Theor. Phys. 123, 89 (2010); N. Ishii et al. (HAL QCD Collaboration), Phys. Lett. B 712, 437 (2012); S. Aoki et al. (HAL QCD Collaboration), Prog. Theor. Exp. Phys. (2012) 01A105.

[4] A. Gezerlis, I. Tews, E. Epelbaum, S. Gandolfi, K. Hebeler, A. Nogga, and A. Schwenk, Phys. Rev. Lett. 111, 032501 (2013).

[5] T. Doi and M. G. Endres, Comput. Phys. Commun. 184, 117 (2013); W. Detmold and K. Orginos, Phys. Rev. D 87, 114512 (2013); J. Günther, B. C. Toth, and L. Varnhorst, ibid. 87, 094513 (2013).

[6] T. Inoue et al. (HAL QCD Collaboration), Phys. Rev. Lett. 106, 162002 (2011).
[7] T. Inoue et al. (HAL QCD Collaboration), Nucl. Phys. A 881, 28 (2012).

[8] T. Inoue et al. (HAL QCD Collaboration), Phys. Rev. Lett. 111, 112503 (2013).

[9] P. Ring and P. Schuck, The Nuclear Many-Body Problem (Springer, Berlin, 1980); G. E. Brown, T. T. S. Kuo et al., The Nucleon-Nucleon Interaction and the Nuclear Many-Body Problem (World Scientific, Singapore, 2010).

[10] S. C. Pieper, Riv. Nuovo Cim. 31, 709 (2008).

[11] P. Navratil, S. Quaglioni, I. Stetcu, and B. R. Barrett, J. Phys. G 36, 083101 (2009)

[12] N. Shimizu, T. Abe, Y. Tsunoda, Y. Utsuno, T. Yoshida, T. Mizusaki, M. Honma, and T. Otsuka, Prog. Theor. Exp. Phys. (2012) 01A205.

[13] G. Hagen, T. Papenbrock, D. J. Dean, and M. Hjorth-Jensen, Phys. Rev. C 82, 034330 (2010).

[14] S. Fujii, R. Okamoto, and K. Suzuki, Phys. Rev. Lett. 103, 182501 (2009). 
[15] W. H. Dickhoff and C. Barbieri, Prog. Part. Nucl. Phys. 52, 377 (2004).

[16] K. Tsukiyama, S. K. Bogner, and A. Schwenk, Phys. Rev. Lett. 106, 222502 (2011).

[17] K. T. R. Davies, M. Baranger, R. M. Tarbutton, and T. T. S. Kuo, Phys. Rev. 177, 1519 (1969).
[18] P. U. Sauer, Nucl. Phys. A 150, 467 (1970).

[19] G. Audi and A. H. Wapstra, Nucl. Phys. A 565, 1 (1993).

[20] K. Murano et al. (HAL QCD Collaboration), Phys. Lett. B 735, 19 (2014).

[21] T. Doi et al. (HAL QCD Collaboration), Prog. Theor. Phys. 127, 723 (2012). 